Journal of Zhejiang University-SCIENCE A (Applied Physics \& Engineering)

ISSN 1673-565X (Print); ISSN 1862-1775 (Online)

www.zju.edu.cn/jzus; www.springerlink.com

E-mail: jzus@zju.edu.cn

\title{
Erratum:
}

\section{Erratum to: Research on the continuous positioning control to servo-pneumatic system}

\author{
Guo-liang $\mathrm{TAO}^{\dagger}$, Xuan-yin WANG, Yong-xiang LU \\ (State Key Laboratory of Fluid Power Transmission and Control, Zhejiang University, Hangzhou 310027, China) \\ †E-mail: gltao@zju.edu.cn
}

doi:10.1631/jzus.2001.e128

Erratum to: J Zhejiang Univ-Sci A (Appl Phys \& Eng) 2001 2(2):128-131 doi:10.1631/jzus.2001.0128

The original version of this article unfortunately contained a mistake. In the second page, before Fig. 2, Eq. (7) was missing, which is given below:

$$
q_{m}=\left\{\begin{array}{l}
\frac{A(u)}{\sqrt{T}} \sqrt{\frac{k}{R} \frac{2}{k-1}} p_{\mathrm{s}} \sqrt{\left(\frac{p}{p_{\mathrm{s}}}\right)^{2 / k}-\left(\frac{p}{p_{\mathrm{s}}}\right)^{(k+1) / k}}, \frac{p}{p_{\mathrm{s}}}>C_{\mathrm{f} 1}, \\
A(u) C_{\mathrm{f} 2}\left(p_{\mathrm{s}}-p\right)^{\lambda}, \frac{p}{p_{\mathrm{s}}} \leq C_{\mathrm{f} 1} .
\end{array}\right.
$$

The online version of the original article can be found at http://dx.doi.org/10.1631/jzus.2001.0128 (C) Zhejiang University and Springer-Verlag Berlin Heidelberg 2013 